\title{
Five and four dimensional experiments for robust backbone resonance assignment of large intrinsically disordered proteins: application to Tau3x protein
}

\author{
Szymon Żerko ${ }^{1}$ Piotr Byrski ${ }^{1}$ - Paweł Włodarczyk-Pruszyński ${ }^{1} \cdot$ Michał Górka $^{1,2}$ • \\ Karin Ledolter $^{3} \cdot$ Eliezer Masliah $^{4} \cdot$ Robert Konrat $^{3} \cdot$ Wiktor Koźmiński $^{1}$
}

Received: 6 April 2016/Accepted: 9 July 2016/Published online: 18 July 2016

(c) The Author(s) 2016. This article is published with open access at Springerlink.com

\begin{abstract}
New experiments dedicated for large IDPs backbone resonance assignment are presented. The most distinctive feature of all described techniques is the employment of MOCCA-XY16 mixing sequences to obtain effective magnetization transfers between carbonyl carbon backbone nuclei. The proposed 4 and 5 dimensional experiments provide a high dispersion of obtained signals making them suitable for use in the case of large IDPs (application to $354 \mathrm{a}$. a. residues of Tau protein $3 \mathrm{x}$ isoform is presented) as well as provide both forward and backward connectivities. What is more, connecting short chains interrupted with proline residues is also possible. All the experiments employ non-uniform sampling.
\end{abstract}

Keywords Intrinsically disordered proteins $\cdot$ Resonance assignment $\cdot$ High-dimensionality NMR $\cdot$ Non-uniform sampling $\cdot$ Isotropic mixing $\cdot$ MOCCA-XY16

Electronic supplementary material The online version of this article (doi:10.1007/s10858-016-0048-7) contains supplementary material, which is available to authorized users.

Wiktor Koźmiński

kozmin@chem.uw.edu.pl

1 Faculty of Chemistry, Biological and Chemical Research Centre, University of Warsaw, 02093 Warsaw, Poland

2 Section of Biophysics, Faculty of Physics, University of Warsaw, 02093 Warsaw, Poland

3 Department of Computational and Structural Biology, Max F. Perutz Laboratories, University of Vienna, Vienna, Austria

4 Departments of Neuroscience and Pathology, University of California, San Diego, La Jolla, CA 92093, USA

\section{Introduction}

Intrinsically disordered proteins (IDPs) or protein regions (IDPRs) have found general interest in the recent molecular biology research (Wright and Dyson 1999; Habchi et al. 2014). These proteins are not only lacking stably folded tertiary structures but also their intrinsic flexibility has significant impact on their biological functionality, therefore challenging the old structurefunction paradigm.

Nuclear magnetic resonance spectroscopy (NMR) is nowadays one of the most efficient spectroscopic techniques in the life sciences, providing insight into molecular structure and dynamics. In recent years, the fast progress of NMR methodology and applications can be observed with special attention paid to difficult, but biologically relevant systems such as IDPs. In contrast to well-folded globular proteins, the peculiar properties of IDPs introduce additional challenges that need to be overcome to obtain spectra enabling effective resonance assignment. The conformational dynamics exhibited by IDPs lead to severe averaging of chemical shifts, which are mostly determined by the amino acid chemical composition and the protein's primary structure. At the same time, however, this dynamic behavior leads to favorable relaxation properties, and allows high dimensionality and long evolution periods within pulse sequences. Prolines are often abundant in IDPs, thus further complicating the resonance assignment procedure.

Application of sparse non-uniform sampling (NUS) in NMR experiments enabled resolution enhancement and high-dimensionality without prohibitively long experiment durations, by the acquisition of only a small fraction of data points required conventionally (Mobli and Hoch 2008; Coggins et al. 2010; Orekhov and Jaravine 2011; Freeman 
and Kupče 2012; Hiller and Wider 2012; Kazimierczuk et al. 2012). In the case of an experiment producing a small number of spectral peaks featuring a limited range of amplitudes, reasonably good spectra can be obtained by employing simple zero-augmented Fourier transformation. However, when artefacts due to incomplete sampling are comparable or stronger than weaker peaks, the spectrum should be reconstructed in order to remove artefacts.

Till now, several strategies were proposed for the reconstruction of sparsely sampled data sets. Among them: maximum entropy (Robin et al. 1991), multidimensional decomposition (MDD) (Orekhov and Jaravine 2011), compressed sensing (CS) (Kazimierczuk and Orekhov 2011; Holland et al. 2011), and signal separation algorithm (SSA) (Stanek and Koźmiński 2010; Stanek et al. 2012). All of them proved their usability for different kinds of experiments.

The approaches for effective resonance assignment of IDPs include automated projection spectroscopy (APSY) (Hiller et al. 2005; Narayanan et al. 2010; Yao et al. 2014), HA (Mäntylahti et al. 2010; Yao et al. 2014) and direct ${ }^{13} \mathrm{C}$ detection (Bermel et al. 2006, 2009; Nováček et al. 2011; Pantoja-Uceda and Santoro 2013), as well as high-dimensional (4-7D) non-uniformly sampled experiments (Kazimierczuk et al. 2013; Nowakowski et al. 2015). In contrast to well folded proteins, where the use of aliphatic ${ }^{1} \mathrm{H}$ and ${ }^{13} \mathrm{C}$ chemical shifts proved their usefulness, in IDPs the aliphatic chemical shifts depend mostly on the residue type. In the case of IDP molecules, the most resolved are the backbone $\mathrm{CO}$ and $\mathrm{N}$ chemical shifts, thus leading to the "CON-CON" approach (Piai et al. 2014; Żerko and Koźmiński 2015).

The aim of present study was to design new techniques capable of providing robust sequential resonance assignment of disordered proteins not only of moderate size but also for larger constructs, as illustrated by a full $\mathrm{CO}, \mathrm{N}$, $\mathrm{N}^{\mathrm{H}}$ resonance assignment of 354 a. a. Tau protein $3 \mathrm{x}$ isoform. To achieve these goals two 5 dimensional: (H)NCOCONH and (HACA)CON(CO)CONH techniques were designed and compared. The use of MOCCA-XY16 block for performing $\mathrm{CO}-\mathrm{CO}$ tocsy mixing as a crucial magnetization transfer between neighbouring amino acid residues was chosen. Both experiments provide robust connectivities through $\mathrm{CO}_{(\mathrm{i}-1)} \mathrm{N}_{(\mathrm{i})}$ chemical shift pairs. 3D HNCO was used as the base spectrum in the Sparse Multidimensional Fourier Transform (Kazimierczuk et al. 2009) processing routine. High dimensionality secures good signal separation while NUS allows to achieve high resolution in indirectly detected dimensions. The first experiment, 5D $(\mathrm{H}) \mathrm{NCOCONH}$, is a direct expansion of a 3 dimensional experiment presented by Yoshimura et al. (2015) who already proved its capability of performing assignment of non-proline resonances of $\alpha$-synuclein. Moreover, he showed that obtaining cross-peaks from $i \pm 3$ residues is possible when long enough mixing time is used. Nevertheless, in our case, it was decided to attune mixing time to provide $i \pm 1$ connectivities as lower number of peaks present in the spectra simplify data analysis as well as leads to a lower sampling noise level. The second experiment, 5D (HACA)CON(CO)$\mathrm{CONH}$, exploits $\mathrm{H} \alpha$ excitation. It improves $\mathrm{S} / \mathrm{N}$ ratio in the case of effectively exchangeable $\mathrm{H}^{\mathrm{N}}$ residues, but more importantly, makes the resonance assignment of proline residues fairly easy. Taking into account the presence of both $i \pm 1$ cross-peaks, it allows reliable resonance assignment of proline abundant protein sequence fragments, which is often the case in IDPs. In comparison to other 5D approaches a significant advantage is the correlation not only with antecedent $(i-1)$ but also with a following $(i+1)$ residue. Aforementioned approach presented by Yoshimura et al. (2015) used only 3D spectra basing on peak separation in the ${ }^{1} \mathrm{H},{ }^{15} \mathrm{~N}$ dimensions. In the figure $\mathrm{S} 1$ the comparison of $3 \mathrm{D}$ and 4D spectra, acquired for Tau3x protein is provided. It clearly shows significantly lower level of peak overlap owing to additional $\mathrm{CO}$ dimension in 4D spectra, which facilitates unambiguous spectra analysis. Another important parameter is the $\mathrm{B}_{0}$ field strength used. Higher field brings improvements in both sensitivity and resolution, on the other hand, this effect is counterbalanced by an increase in relaxation loses during the $\mathrm{CO}$ evolution and mixing periods. In fact, MOCCA-XY16 mixing sequence minimizes relaxation loses (Furrer et al. 2004) since the magnetization is transverse only during $180^{\circ}$ pulses. Nevertheless, such relaxation loses become significant for long mixing periods. Since relaxation of $\mathrm{CO}$ nuclei is dominated by the chemical shift anisotropy mechanism, the effectiveness of which scales up with the square of $\mathrm{B}_{0}$, the use of higher $\mathrm{B}_{0}$ fields may not be an optimal choice. Hence, proposed pulse sequences' performance was tested on 600 and $800 \mathrm{MHz}$ spectrometers.

In principle, the resonance assignment strategy based on a 3D HNCO and a single 5D experiment (HACA)$\mathrm{CON}(\mathrm{CO}) \mathrm{CONH}$ could provide complete (including proline residues) and robust data for a backbone $\left(\mathrm{H}^{\mathrm{N}}, \mathrm{N}\right.$ and $\mathrm{CO})$ resonance assignment. The application to Tau3x isoform proved that this ideal case in not as far from reality as one could imagine. First, application of both 5D experiments to a sample of $\alpha$-synuclein, a medium size (140 a.a.) IDP is presented. Finally, application of two complementary 4D experiments derived from 5D (HACA)CON(CO)CONH performed on a Tau $3 \mathrm{x}$ protein sample is discussed and compared with results obtained using a different set of $5 \mathrm{D}$ experiments. 


\section{Materials and methods}

\section{Pulse sequences}

Magnetization transfers schemes and pulse sequences of 5D (H)NCOCONH and 5D (HACA)CON(CO)CONH are provided in the Figs. 1 and 2. The radiofrequency (RF) field of the CO-selective $180^{\circ}$ pulses in MOCCA-XY16 was set at $4.68(6.23) \mathrm{kHz}$ with a duration of the $180^{\circ}$ pulse $(d)$ of 107.0 (80.2) $\mu$ s on the 600 (800) $\mathrm{MHz}$ spectrometer, which was determined in order to ensure that $\mathrm{C} \alpha$ nuclei experience an effective $720^{\circ}$ rotation during each carbonyl $180^{\circ}$ pulse (Felli et al. 2009). It is possible to choose pulse power to get $360^{\circ}$ rotation for $\mathrm{C} \alpha$ but it would significantly increase the amount of energy dissipated at the RF coil. Furthermore, $720^{\circ}$ rotation choice leads to narrower inversion profiles but in the case of IDPs such narrow bandwidths are still sufficient to cover all involved nuclei. Value of the $180^{\circ}$ pulse repetition period $\Delta$ ( $\Delta$ is calculated including $180^{\circ}$ pulse length) was initially set to $500 \mu \mathrm{s}$. Mixing time was attuned to provide magnetizations transfers between consecutive $\mathrm{CO}$ nuclei securing the presence of $i \pm 1$ cross-peaks.

\section{NMR spectroscopy}

All measurements were performed on Agilent DD2 600 and $800 \mathrm{MHz}$ spectrometers equipped with standard triple resonance room temperature probes. In all experiments: four scans were acquired for each FID, relaxation delay of $1.3 \mathrm{~s}$ was used, mixing time was set to $250 \mathrm{~ms}$ and temperature to $288 \mathrm{~K}$. $\Delta$ of 500 and $250 \mu$ s was used for $\alpha$ synuclein and Tau3x protein measurements, respectively. Detailed spectral widths and maximal evolution times are given in the Supporting Information Table S1. The pulse sequence codes for Varian/Agilent spectrometers are available from the authors on demand.

For 5D spectra 3D HNCO was used as an input for Sparse Multidimensional Fourier Transform procedure (Kazimierczuk et al. 2009). All spectra were processed using ToASTD (Kazimierczuk et al. 2006), additionally cleaner3d (Stanek and Koźmiński 2010), cleaner4d (Stanek et al. 2012), and reduced (Kazimierczuk et al. 2009) programmes were used for processing 3D, 4D and 5D spectra, respectively. All used processing software is available at http://nmr.cent3.uw.edu. $\mathrm{pl} /$ software. All spectra were inspected using the Sparky program (Goddard and Kneller 2000), Nmrglue Python package was used for visualization purposes (Helmus and Jaroniec 2013). The whole spectra analysis and resonance assignment were performed manually.

\section{Sample preparation}

NMR sample of $\alpha$-synuclein contained $1 \mathrm{mM}$ of ${ }^{13} \mathrm{C}$, ${ }^{15} \mathrm{~N}$-labeled $\alpha$-synuclein in a $20 \mathrm{mM}$ sodium phosphate buffer, $\mathrm{pH}$ 6.5, $200 \mathrm{mM} \mathrm{NaCl}, 10 \% \mathrm{D}_{2} \mathrm{O}$.

NMR sample of Tau $3 \mathrm{x}$ contained $0.5 \mathrm{mM}$ of ${ }^{13} \mathrm{C}$, ${ }^{15} \mathrm{~N}$-labeled Tau3x in $20 \mathrm{mM}$ NAP, $1 \mathrm{mM} \beta$-Merc.EtOH, $1 \mathrm{mM}$ EDTA, $1 \mathrm{mM} \mathrm{NaN}_{3}, 100 \mathrm{mM} \mathrm{NaCl}, 10 \% \mathrm{D}_{2} \mathrm{O}$.

\section{Results and discussion}

\section{Application to $\alpha$-synuclein}

A crucial acquisition parameter is the length of the mixing sequence. To find an efficient duration of the mixing time
Fig. 1 Coherence pathway schemes for $5 \mathrm{D}(\mathrm{H}) \mathrm{NCOCONH}$ (top) and 5D

(HACA)CON(CO)CONH

(bottom) experiments

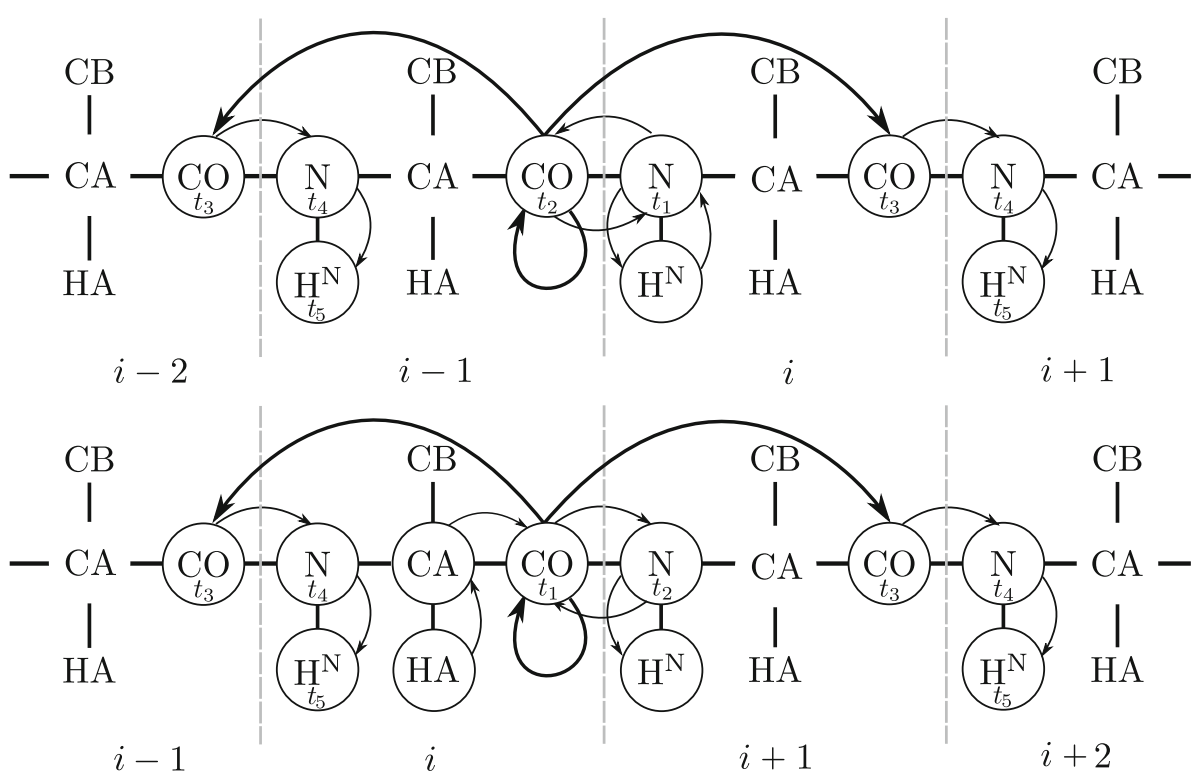




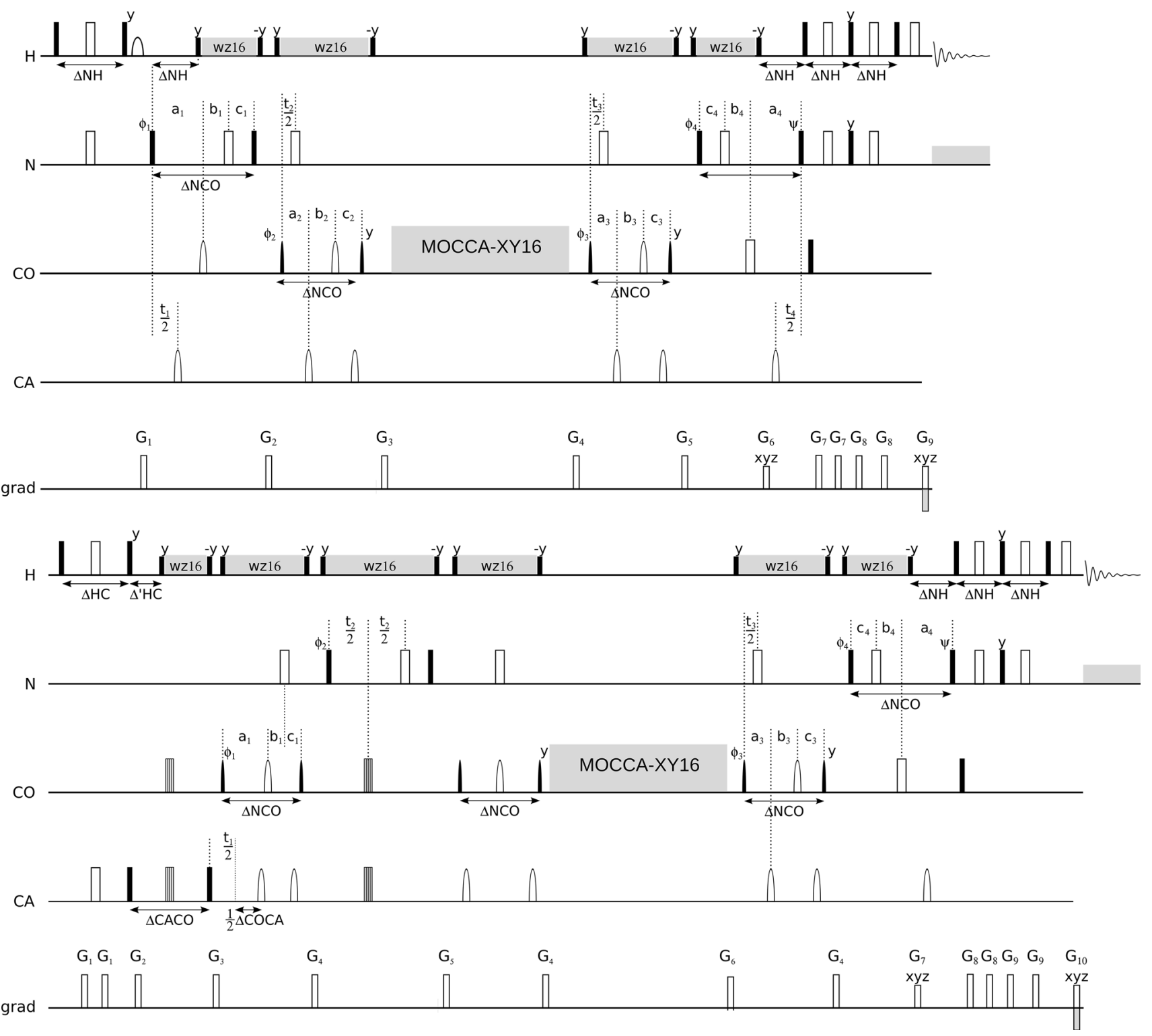

Fig. 2 (H)NCOCONH (top) and (HACA)CON(CO)CONH (bottom) experiments pulse sequence schemes. Rectangles represent hard pulses. Filled and empty symbols represent $90^{\circ}$ and $180^{\circ}$ pulses, respectively. ${ }^{1} \mathrm{H}$ and ${ }^{15} \mathrm{~N}$ composite pulse decoupling is performed with WALTZ-16 (Shaka et al. 1983), at $\gamma \mathrm{B}_{1} / 2 \pi$ of 5.1 and $1.14 \mathrm{kHz}$ at $800 \mathrm{MHz}$ spectrometer, and 3.37 and $0.97 \mathrm{kHz}$ at $600 \mathrm{MHz}$, respectively. Simultaneous inversion of CA and CO spins is achieved using a 6-element composite pulse (Shaka 1985). Selective CA and CO ${ }^{13} \mathrm{C}$ pulses are applied with the RF field strength adjusted to $\left|\Delta \Omega_{\mathrm{CA}}-\mathrm{CO}\right| /$ $\sqrt{ } 15(\sqrt{ } 3)$ for $90^{\circ}$ and $180^{\circ}$ pulses, respectively. For the $800 \mathrm{MHz}$ spectrometer $90^{\circ}$ and $180^{\circ}$, rectangular and sinc-shaped pulsed (bellshaped at the scheme) with a duration of 40.1 (35.9) $\mu$ s, and 65.8 (58.8) $\mu$ s, respectively, are used. Whereas, for the $600 \mathrm{MHz}$ spectrometer the respective pulse lengths are 53.5 (47.9) $\mu$ s and 87.8 (78.4) $\mu$ s. Off-resonance pulses are applied using phase modulation of the carrier. The PFG durations are set to $0.5 \mathrm{~ms}$, except for coherence selection gradients for which 2.0 and $0.2 \mathrm{~ms}$, are used. Delay durations are set as follows: $\Delta \mathrm{NH}=5.4 \mathrm{~ms}, \Delta \mathrm{NCO}=28 \mathrm{~ms}$, $\Delta \mathrm{COCA}=9.1 \mathrm{~ms}$. Evolution periods for $\mathrm{H}, \mathrm{N}, \mathrm{CO}$ are in semiconstant-time mode: $\quad a_{i}=\left(t_{i}+\Delta\right) / 2 ; \quad b_{i}=t_{i}\left(1-\Delta / t_{\text {imax }}\right) / 2$; $c_{i}=\Delta\left(1-t_{i} / t_{\text {imax }}\right) / 2$ or in constant-time mode: $a_{i}=\left(t_{i}+\Delta\right) / 2$; $\mathrm{b}_{\mathrm{i}}=0 ; \mathrm{c}_{\mathrm{i}}=\left(\Delta-\mathrm{t}_{\mathrm{i}}\right) / 2$ where $\Delta$ stands for $\Delta \mathrm{NH}, \Delta \mathrm{NCO}$. In the second sequence evolution for $\mathrm{N}$ in $\mathrm{t}_{2}$ is in real-time mode. The four step phase cycle is: $\phi_{1}=\mathrm{x},-\mathrm{x} ; \phi_{2}=2 \mathrm{x}, 2(-\mathrm{x})$ and $\phi_{\mathrm{rec}}=\mathrm{x}$, $2(-x), x$. In $t_{1}, t_{2}, t_{3}, t_{4}$ dimensions quadrature is accomplished using States-TPPI method, by incrementing $\phi_{1}, \phi_{2}, \phi_{3}, \phi_{4}$ phases, respectively. The phase $\psi=\mathrm{x}$ is inverted simultaneously with the last gradient pulse to achieve echo-antiecho coherence transfer selection in the last indirect dimension. The coherence selection gradients marked with $x y z$ are applied at the magic angle $(600 \mathrm{MHz})$ or along the $\mathrm{z}$ axis $(800 \mathrm{MHz}) .180^{\circ}$ water $1.42(1.07) \mathrm{ms}$ sinc-shaped flipback pulses are used, on the 600 and $800 \mathrm{MHz}$ spectrometers, respectively 
two $(\mathrm{H}) \mathrm{NCOCONH}$ spectra were recorded with mixing times set to 150 and $250 \mathrm{~ms}$, respectively. It appears that for the employed MOCCA-XY16 implementation longer mixing times are preferred (see columns 1 and 2 in the Table 1) and $250 \mathrm{~ms}$ mixing time was used for all further acquisitions. Since, the rate of coherence transfer does not depend on the field strength, the value established at $800 \mathrm{MHz}$, due to better relaxation properties at lower field, is always a safe choice. The next step of the study was to compare the performance of the two proposed pulse sequences: (H)NCOCONH and (HACA)CON(CO)CONH (see Fig. 3 for the examples of obtained 2D cross-sections).
Table 1 Comparison of results obtained with different parameters for $5 \mathrm{D}$

(H)NCOCONH and 5D (HACA)CON(CO)CONH experiments for the $\alpha$-synuclein sample

\begin{tabular}{llll}
\hline & 1 & 2 & 3 \\
\hline Backwards links & $25 \%(32 / 128)$ & $77 \%(99 / 128)$ & $79 \%(105 / 133)$ \\
Forward links & $22 \%(28 / 128)$ & $71 \%(91 / 128)$ & $79 \%(105 / 133)$ \\
Number of points & 1100 & 1100 & 1100 \\
Mixing time (ms) & 150 & 250 & 250 \\
Spectrometer $(\mathrm{MHz})$ & 800 & 800 & 800 \\
Pulse sequence & $(\mathrm{H}) \mathrm{NCOCONH}$ & $(\mathrm{H}) \mathrm{NCOCONH}$ & $(\mathrm{HACA}) \mathrm{CON}(\mathrm{CO}) \mathrm{CONH}$ \\
\hline
\end{tabular}

5D (H)NCOCONH
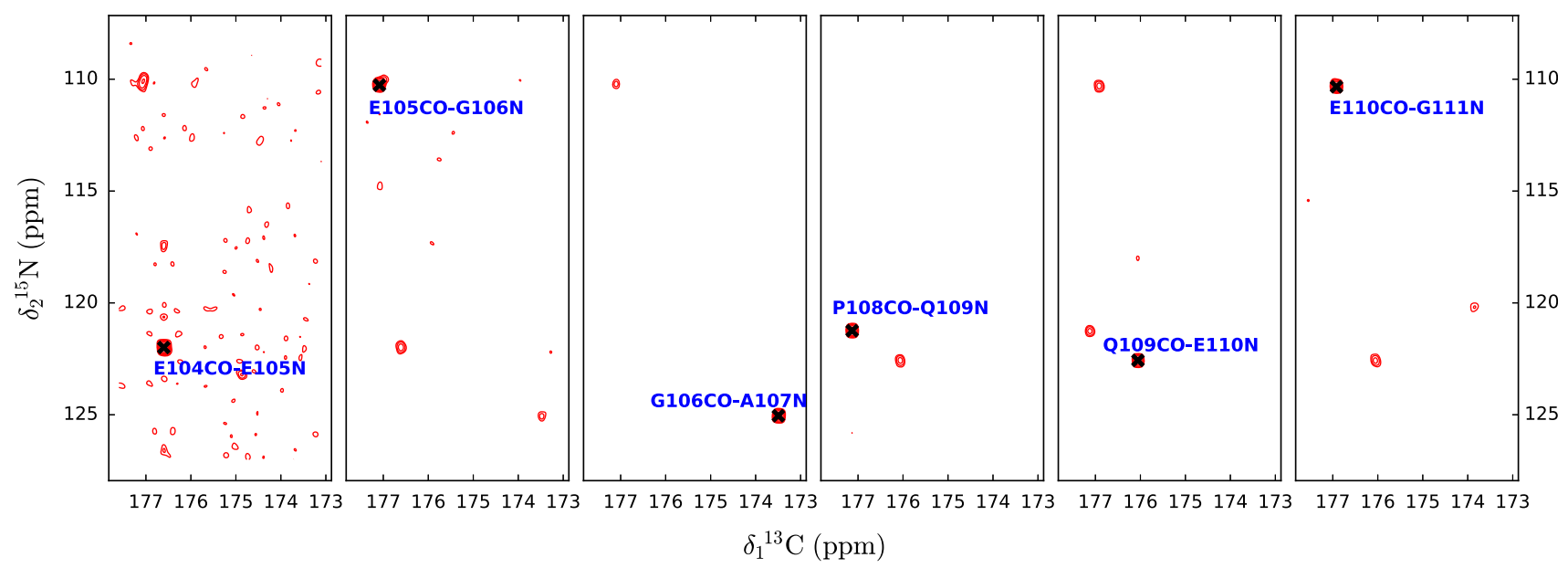

5D (HACA)CON(CO)CONH
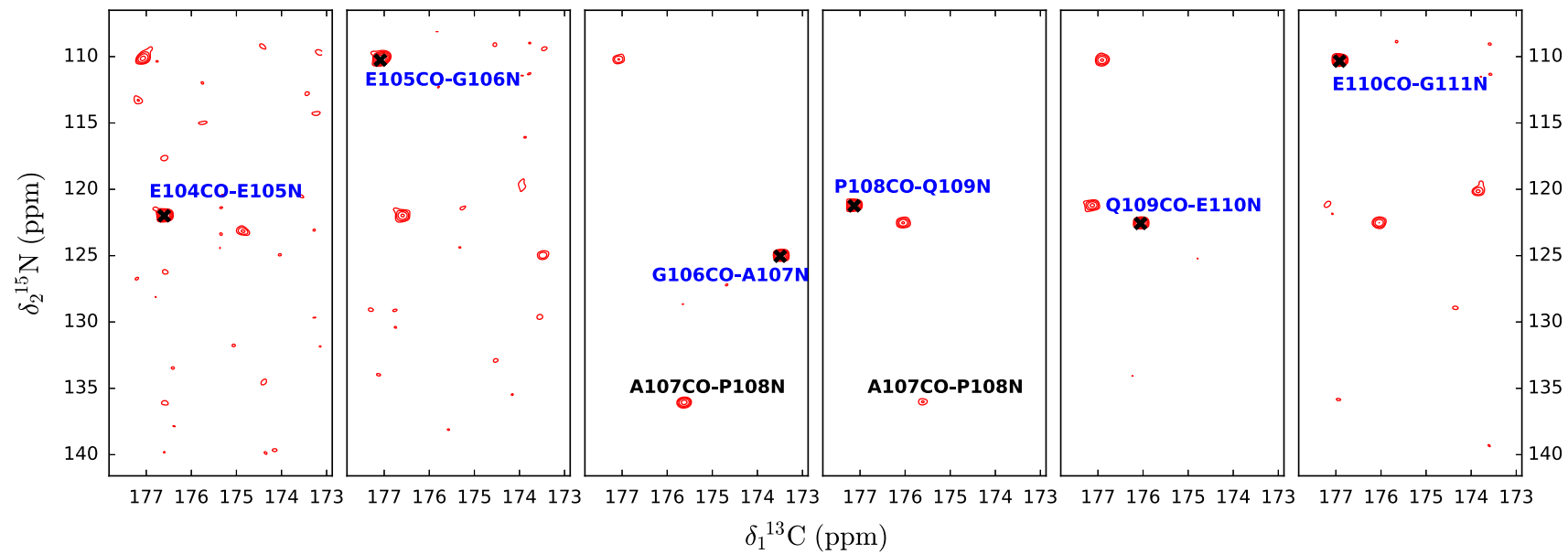

$\begin{array}{llllllllll}177 & 176 & 175 & 174 & 173 & 177 & 176 & 175 & 174 & 173\end{array}$
Fig. 3 Resulting 2D cross-sections from 5D (H)NCOCONH (top) and 5D (HACA)CON(C)CONH for residues $105-111$ of $\alpha$-synuclein (strip plot for P108 is not shown). Diagonal peak positions are marked with black crosses. Note the presence of cross-peaks A107CO-P108N on 3rd and 4th panels (bottom), which allow sequential assignment despite the presence of a proline residue 
Note that, since $\mathrm{S} / \mathrm{N}$ ratio in the case of non-uniformly sampled spectra is an ill-defined parameter (Hyberts et al. 2013), to objectively evaluate the results from different acquisition setups the number of identified peaks was compared. As one can see from comparison of the results presented in the columns 2 and 3, Table 1. (HACA)$\mathrm{CON}(\mathrm{CO}) \mathrm{CONH}$ technique provides slightly more complete results. Note, that this experiment additionally allows effective resonance assignment of proline residues. The smaller influence of the $\mathrm{H}^{\mathrm{N}}$ exchange on the performance of $5 \mathrm{D}$ (HACA)CON(CO)CONH seems to counterbalance the use of a longer and more complicated pulse sequence.

To finally evaluate the performance of the proposed (HACA)CON(CO)CONH experiment, two longer $(71 \mathrm{~h}$ long each) acquisitions were performed on two different spectrometers, 600 and $800 \mathrm{MHz}$ (see Table 2). Although the results of the measurements on both spectrometers were comparable, surprisingly, the lower $(600 \mathrm{MHz})$ field spectrometer provided slightly more information.

\section{Application to Tau3x isoform}

The resonance assignment of Tau3x isoform was first attempted using a set of experiments: 3D HNCO, 5D $\mathrm{HN}(\mathrm{CA}) \mathrm{CONH}$ (Kazimierczuk et al. 2010) and 5D (HACA)CON(CA)CONH (Zawadzka-Kazimierczuk et al. 2012). Additionally, for easy amino acid residue type determination 5D HabCabCONH (Kazimierczuk et al. 2010) and 5D HC(CC-TOCSY)CONH (Kazimierczuk et al. 2009; Hiller et al. 2008) were recorded. All spectra were recorded using a $800 \mathrm{MHz}$ spectrometer equipped with a standard triple resonance room-temperature probe, total measurement time for all 5D experiments was $92 \mathrm{~h}$. The obtained results are depicted in Fig. 4. As one can see, the vast majority of the residues have been assigned (328 out of $353 \mathrm{CO}_{\mathrm{i}-1} \mathrm{~N}_{\mathrm{i}}$ pairs) which would ordinarily be considered a satisfactory result. Nevertheless, all of the unassigned $\mathrm{CO}_{\mathrm{i}-1} \mathrm{~N}_{\mathrm{i}}$ pairs are located in proline rich fragments of Tau3x protein sequence, clearly showing that the assignment procedure was limited by insufficient information gained from analysed spectra. In other words, for a full assignment of such regions a different approach is needed.

Knowing that sampling artefacts will be even more troublesome in the case of a larger protein and taking into account the incomplete set of signals obtained for the $\alpha$ synuclein sample, suggesting limited sensitivity of the proposed techniques, it was decided to lower the dimensionality of experiments used for Tau3x resonance assignment. Application of 4D spectra allows access to a vast number of efficient reconstruction methods, which suppress sampling artefacts (i.e. Maximum entropy, signal separation algorithm). In addition, 4D experiments are more sensitive, on the other hand they provide more ambiguous data. Naturally, different choice of 4 from 5 dimensions in a 4D experiment derived from the proposed $5 \mathrm{D}$ experiment is possible. In our approach each 4D experiment provides only one new chemical shift ( $\mathrm{CO}$ or $\mathrm{N})$ correlating it with the three chemical shifts already known from 3D HNCO-we call this the " $1+3$ " approach. Obviously several " $2+2$ " $4 \mathrm{D}$ experiments could be proposed (i.e. $\mathrm{CON}+\mathrm{H}^{\mathrm{N}}, \mathrm{N}$; $\mathrm{CON}+\mathrm{H}^{\mathrm{N}}, \mathrm{CO}$;). We concluded that all of these " $2+2$ " possibilities are less favourable, mainly because the main bottleneck in the
Table 2 Comparison of results obtained from $5 \mathrm{D}$ (HACA)CON(CO)CONH experiment using 600 and $800 \mathrm{MHz}$ spectrometers for the $\alpha$-synuclein sample

\begin{tabular}{lll}
\hline & 1 & 2 \\
\hline Backwards links & $90 \%(120 / 133)$ & $92 \%(123 / 133)$ \\
Forward links & $88 \%(117 / 133)$ & $94 \%(125 / 133)$ \\
Number of points & 2250 & 2250 \\
Mixing time (ms) & 250 & 250 \\
Spectrometer $(\mathrm{MHz})$ & 800 & 600 \\
Pulse sequence & (HACA)CON(CO)CONH & (HACA)CON(CO)CONH
\end{tabular}

\section{GAMAEPRQEF EVMEDHAGTY GLGDRKDQGG YTMHQDQEGD TDAGLKAEEA GIGDTPSLED EAAGHVTQAR MVSKSKDGTG SDDKKAKGAD GKTKIATPRG AAPPGQKGQA NATRIPAKTP PAPKTPPSSG EPPKSGDRSG YSSPGSPGTP GSRSRTPSLP TPPTREPKKV AVVRTPPKSP SSAKSRLQTA PVPMPDLKNV KSKIGSTENL KHQPGGGKVQ IVYKPVDLSK VTSKCGSLGN IHHKPGGGQV EVKSEKLDFK DRVQSKIGSL DNITHVPGGG NKKIEGHKLT FRENAKAKTD HGAEIVYKSP VVSGDTSPRH LSNVSSTGSI DMVDSPQLAT LADEVSASLA KQGL}

Fig. 4 Tau3x protein sequence with resonance assignment of CON pairs obtained using: 3D $\mathrm{HNCO}, 5 \mathrm{D} \mathrm{HN}(\mathrm{CA}) \mathrm{CONH}, 5 \mathrm{D}$ (HACA)$\mathrm{CON}(\mathrm{CA}) \mathrm{CONH}, 5 \mathrm{Dn} \mathrm{HabCabCONH}$ and $5 \mathrm{D} \mathrm{HC}(\mathrm{CC}-$
TOCSY)CONH. Green CON pair assigned, black only $\mathrm{CO}$ assigned, blue only $\mathrm{N}$ assigned, red no assignment 
analysis of resultant spectra of all of them would be the fact that none of $2 \mathrm{D}$ correlations could provide sufficiently dispersed peaks. Even the most desired $\mathrm{CO}_{(\mathrm{i}-1)} \mathrm{N}_{(\mathrm{i})}$ correlation 2D spectrum becomes crowded in the case of relatively big proteins (see Fig. 5) and providing additional $\mathrm{H}^{\mathrm{N}}$ chemical shift can significantly reduce this problem. Another drawback of a $\mathrm{CON}+\mathrm{COH}^{\mathrm{N}}$ approach would be the lack of sensitivity enhancement that would compromise the signal to noise ratio in such a $4 \mathrm{D}$ experiment.

The second attempt used only the 3D HNCO, and two complementary 4D spectra: (HACA)CO(NCO)CONH and (HACACO)N(CO)CONH (66 h long each). All spectra were recorded using a $600 \mathrm{MHz}$ spectrometer equipped with a standard triple resonance room-temperature probe. The 4D experiments used correlate $\mathrm{CO}_{(\mathrm{i}-1)}$ and $\mathrm{N}$ (i) chemical shifts with signals present in the 3D HNCO. As a result, 4D spectra can be inspected in a manner similar to the usual 3D triple resonance experiments used for protein sequential resonance assignment (see Figs. 6, 7). The only difference is that instead of ${ }^{1} \mathrm{H},{ }^{15} \mathrm{~N}-\mathrm{HSQC}$ a $3 \mathrm{D}$
HNCO is treated as a base for the analysis of strip plots obtained from 4D experiments, which allows for a disambiguation of spectra analysis, as the resonance assignment process of IDPs is hampered by a severe peak overlap (see figure $\mathrm{S} 1$ for a comparison of peaks separation provided by the used 4D experiments with 3D (HACACO)N(CO$\mathrm{CO}) \mathrm{NH}$ and 3D (HACA)CO(NCOCO)NH). Both 4D spectra were processed using the cleaner4d program (Stanek et al. 2012) performing spectral reconstruction using the SSA algorithm, currently the only one capable of efficient processing of such high resolution 4D data sets, with file sizes reaching 140 GBs. What is worth mentioning, no additional spectra providing $C \alpha$ and/or $C \beta$ chemical shifts were used to aid with the identification of residue type, as it was not found to be necessary. Since a $600 \mathrm{MHz}$ spectrometer was used, relaxation loses during tocsy mixing were significantly suppressed in comparison to the $800 \mathrm{MHz}$ spectrometer, and to improve mixing effectiveness taking advantage of lower power load at $600 \mathrm{MHz}$, it was decided to use a shorter $\Delta$ value of $250 \mu$ s.

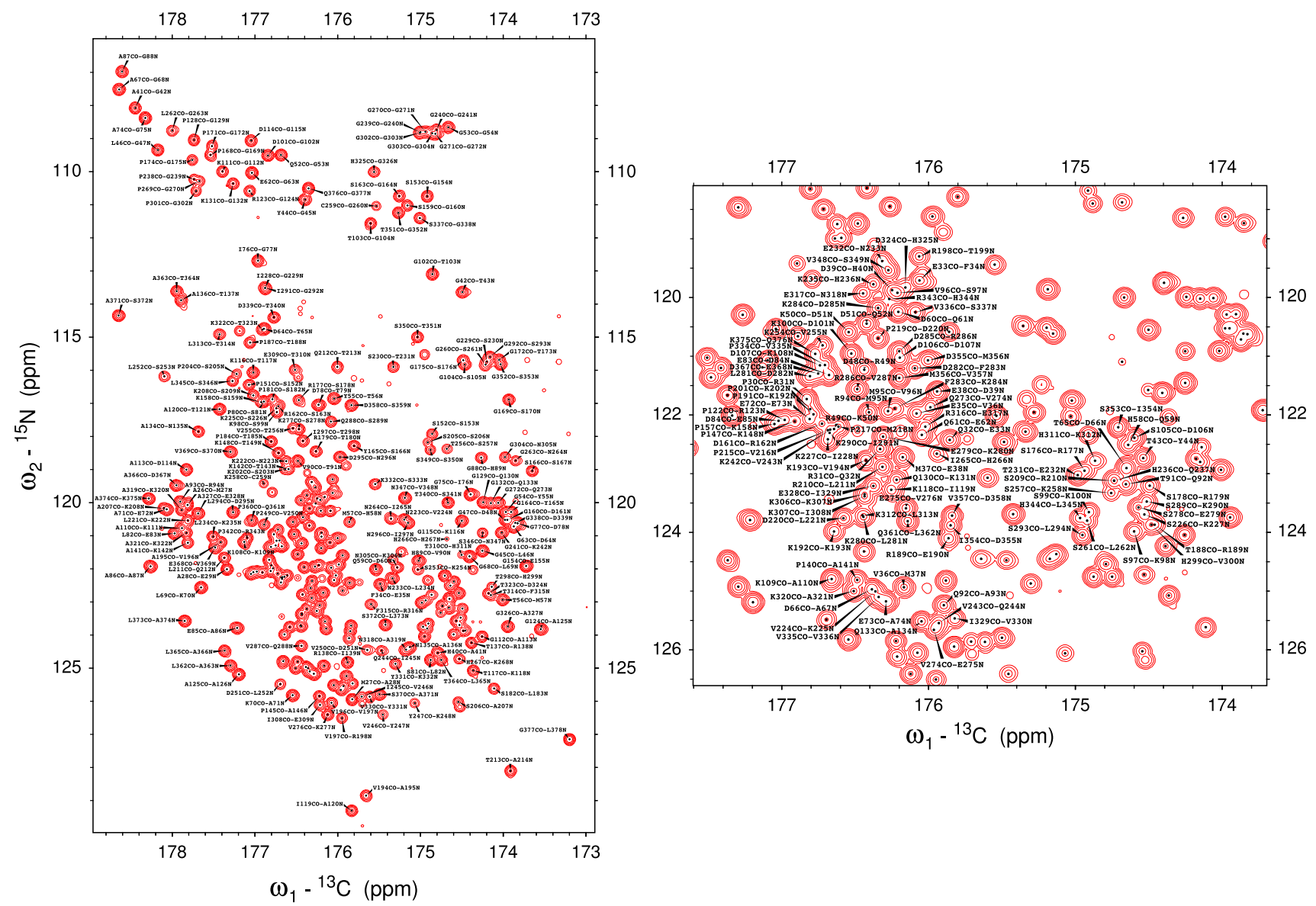

Fig. 5 Tau3x CON projection taken from a 3D HNCO spectrum (left). Zoom into the most crowded part of the CON projection (right) 


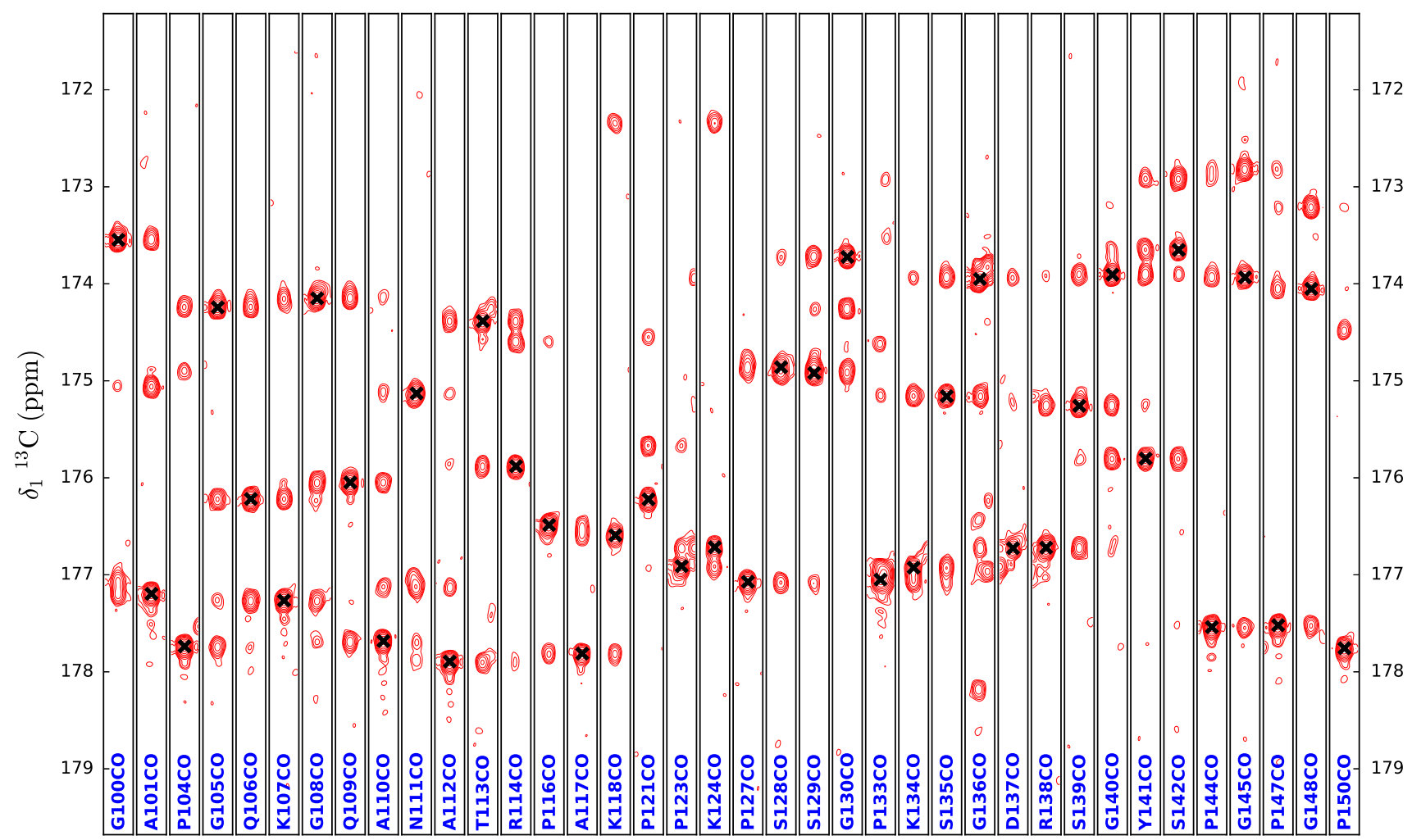

Fig. 6 Strip plots for the residues from A101 to G151 taken from 4D (HACA)CO(NCO)CONH of Tau3x. Diagonal peak positions are marked with black crosses. Strips plots for proline residues are not shown. ${ }^{13} \mathrm{C}$ chemical shifts of residues preceding prolines are evaluated from cross-peaks present on neighbouring residues' strip plots
The final results are depicted in Fig. 8. Only $1 \mathrm{G}$ and 150P N and 354L CO chemical shifts are missing. Additionally, the mixed-up assignments of 213-214 and 276-277 residues were identified and corrected. Overall performance of the strategy used is more than satisfactory, as an almost full assignment was obtained, even though the experiments were performed using a standard $600 \mathrm{MHz}$ spectrometer and without the use of an expensive cryogenically cooled probe.

\section{Discussion}

The backbone $\left(\mathrm{H}^{\mathrm{N}}, \mathrm{N}\right.$ and $\left.\mathrm{CO}\right)$ resonance assignment obtained with (4)5D (HACA)CON(CO)CONH could be easily complemented with $\mathrm{C} \alpha / \beta$ and $\mathrm{H} \alpha / \beta$ chemical shifts, for example with 5D HabCabCONH. It would be even possible to incorporate evolutions of CA and HA chemical shifts within the presented experiments in a similar manner to the recently presented hybrid of sparse random sampling and projection spectroscopy (Żerko and Koźmiński 2015). Resonance assignment can be further expanded to the side chains using 5D HC(CC-TOCSY)CONH leading to a full signal assignment.

In our study of Tau3x isoform, 5D (HACA)CON(CO)$\mathrm{CONH}$ did not provide sufficient sensitivity. It should be possible to overcome this limitation by utilising a cryogenically cooled probe, especially employing shaped salt tolerant NMR tubes. Spectral quality would surely benefit from the removal of sampling artefacts which at present is not possible for 5 dimensional spectra.

Results obtained from a set of two 4D spectra are more than satisfactory. In similar challenging studies of big disordered proteins comparably complete resonance assignment was obtained only for the MAP2c protein (Nováček et al. 2013). Previous studies of proteins from the Tau family provided less complete assignments than this study (Narayanan et al. 2010; Yao et al. 2014). What is more, all of aforementioned studies were performed on significantly more concentrated samples and using far more expensive high-field spectrometers equipped with cryogenically cooled probes, which demonstrates the superiority of the novel approach presented herein. 


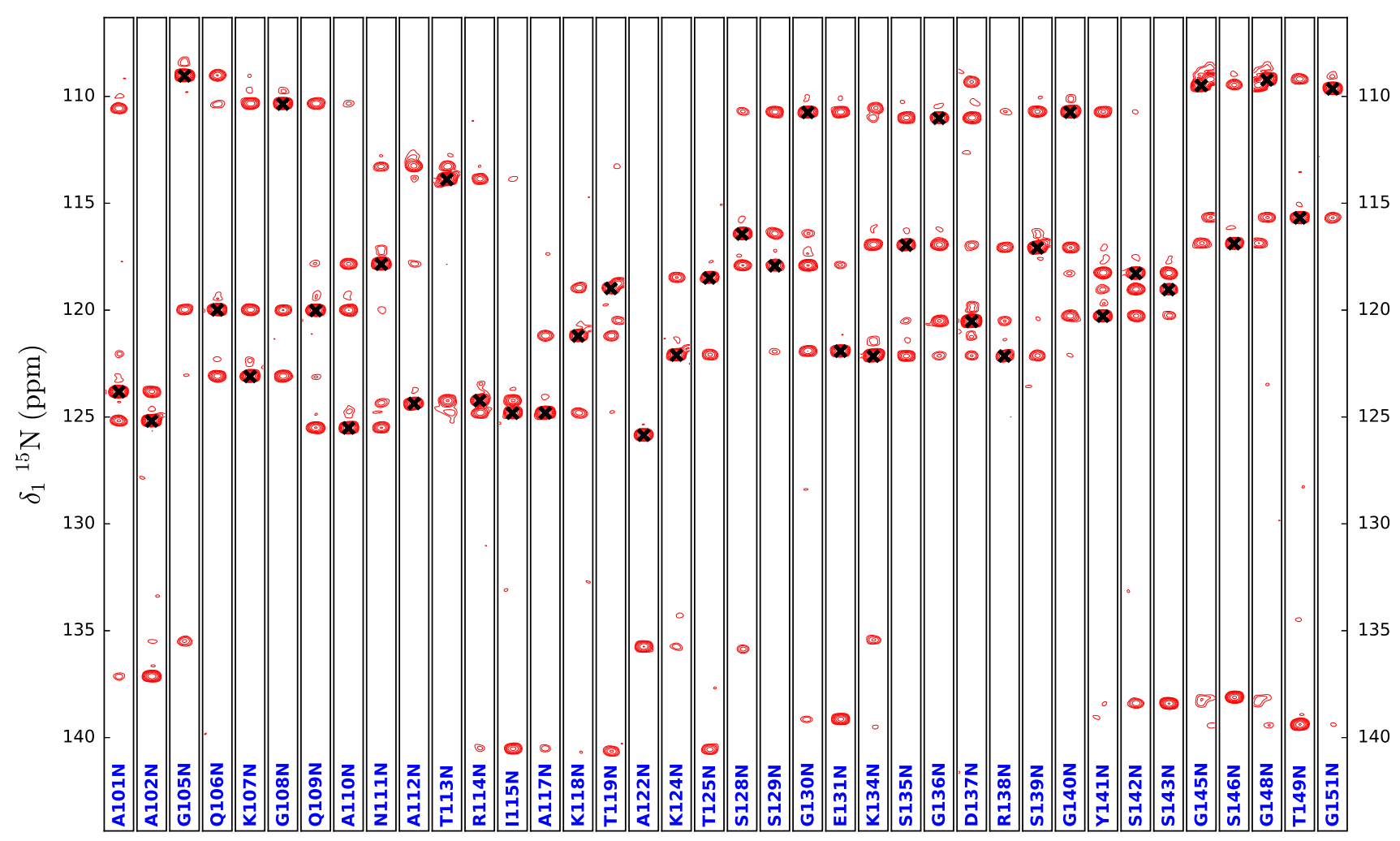

Fig. 7 Strip plots for the residues from A101 to G151 taken from 4D (HACACO)N(CO)CONH of Tau3x. Diagonal peak positions are marked with black crosses. Strips plots for proline residues are not shown, nonetheless their ${ }^{15} \mathrm{~N}$ chemical shifts are evaluated from cross-peaks present on neighbouring residues' strip plots. The small, lower ${ }^{15} \mathrm{~N}$ frequency peaks originate from ${ }^{2} \mathrm{H}^{15} \mathrm{~N}-{ }^{1} \mathrm{H}^{15} \mathrm{~N}$ isotopomers

\section{GAMAEPRQEF EVMEDHAGTY GLGDRKDQGG YTMHQDQEGD TDAGLKAEEA GIGDTPSLED EAAGHVTQAR MVSKSKDGTG SDDKKAKGAD GKTKIATPRG AAPPGQKGQA NATRIPAKTP PAPKTPPSSG EPPKSGDRSG YSSPGSPGTP GSRSRTPSLP TPPTREPKKV AVVRTPPKSP SSAKSRLQTA PVPMPDLKNV KSKIGSTENL KH GGGKVQ IVYKPVDLSK VTSKCGSLGN IHHKPGGGQV EVKSEKLDFK DRVQSKIGSL DNITH_GGG NKKIEGHKLT FRENAKAKTD HGAEIVYKSP VVSGDTSPRH LSNVSSTGSI DMVDSPQLAT LADEVSASLA KQGL}

Fig. 8 Tau3x protein sequence with resonance assignment of CON pairs obtained using 3D $\mathrm{HNCO}, 4 \mathrm{D}$ (HACA)CO(NCO)CONH and 4D (HACACO)N(CO)CONH. Green $\mathrm{CON}$ pair assigned, black only

\section{Conclusions}

We presented new 4D and 5D experiments for the backbone resonance assignment of intrinsically disordered proteins. Described techniques use CO-CO tocsy type magnetization transfers and are especially effective in the resonance assignment of proline rich sequence fragments. We proved that these experiments can be successfully run on a standard room-temperature probe, even on a medium field NMR spectrometer $(600 \mathrm{MHz})$.

Acknowledgments This work was supported by the Polish National Science Centre with PRELUDIUM Grant No. 2013/11/N/ST4/01832. The study was carried out at the Biological and Chemical Research Centre, University of Warsaw, established within a project co-financed by the European Union from the European Regional
CO assigned, blue only $\mathrm{N}$ assigned. Red background indicates correction of a previous assignment

Development Fund under the Operational Programme Innovative Economy, 2007-2013.

Open Access This article is distributed under the terms of the Creative Commons Attribution 4.0 International License (http://crea tivecommons.org/licenses/by/4.0/), which permits unrestricted use, distribution, and reproduction in any medium, provided you give appropriate credit to the original author(s) and the source, provide a link to the Creative Commons license, and indicate if changes were made.

\section{References}

Bermel W, Bertini I, Felli IC, Lee Y, Luchinat C, Pierattelli R (2006) Protonless NMR experiments for sequence-specific assignment of backbone nuclei in unfolded proteins. J Am Chem Soc 128:3918-3919. doi:10.1021/ja0582206 
Bermel W, Bertini I, Felli IC, Pierattelli R (2009) Speeding up ${ }^{13} \mathrm{C}$ direct detection biomolecular NMR spectroscopy. J Am Chem Soc 131:15339-15345. doi:10.1021/ja9058525

Coggins BE, Venters RA, Zhou P (2010) Radial sampling for fast NMR: concepts and practices over three decades. Prog Nucl Magn Reson Spectrosc 57:381-419. doi:10.1016/j.pnmrs.2010. 07.001

Felli IC, Pierattelli R, Glaser SJ, Luy B (2009) Relaxation-optimised Hartmann-Hahn transfer using a specifically Tailored MOCCAXY16 mixing sequence for carbonyl-carbonyl correlation spectroscopy in 13C direct detection NMR experiments. J Biomol NMR 43:187-196. doi:10.1007/s10858-009-9302-6

Freeman R, Kupče E (2012) Concepts in projection-reconstruction. Top Curr Chem 316:1-20. doi:10.1007/128

Furrer J, Kramer F, Marino JP, Glaser SJ, Luy B (2004) Homonuclear Hartmann-Hahn transfer with reduced relaxation losses by use of the MOCCA-XY16 multiple pulse sequence. J Magn Reson 166:39-46

Goddard TD, Kneller DG (2000) SPARKY 3. University of California, San Francisco

Habchi J, Tompa P, Longhi S, Uversky VN (2014) Introducing protein intrinsic disorder. Chem Rev 114:6561-6588. doi:10. $1021 / \mathrm{cr} 400514 \mathrm{~h}$

Helmus JJ, Jaroniec CP (2013) Nmrglue: an open source python package for the analysis of multidimensional NMR data. J Biomol NMR 55:355-367. doi:10.1007/s10858-013-9718-x

Hiller S, Wider G (2012) Automated projection spectroscopy and its applications. Top Curr Chem 316:21-47. doi:10.1007/128

Hiller S, Fiorito F, Wüthrich K, Wider G (2005) Automated projection spectroscopy (APSY). Proc Natl Acad Sci USA 102:10876-10881. doi:10.1073/pnas.0504818102

Hiller S, Joss R, Wider G (2008) Automated NMR assignment of protein side chain resonances using automated projection spectroscopy (APSY) experiments. JACS 130:12073-12079. doi:10.1021/ja803161d

Holland DJ, Bostock MJ, Gladden LF, Nietlispach D (2011) Fast multidimensional NMR spectroscopy using compressed sensing. Angew Chem Int Ed Engl 50:6548-6551. doi:10.1002/anie. 201100440

Hyberts S, Robson S, Wagner G (2013) Exploring signal-to-noise ratio and sensitivity in non-uniformly sampled multi-dimensional NMR spectra. J Biomol NMR 55:167-178. doi:10.1007/ s10858-012-9698-2

Kazimierczuk K, Orekhov VY (2011) Accelerated NMR spectroscopy by using compressed sensing. Angew Chem Int Ed Engl 50:5556-5559. doi:10.1002/anie.201100370

Kazimierczuk K, Zawadzka A, Koźmiński W, Zhukov I (2006) Random sampling of evolution time space and Fourier transform processing. J Biomol NMR 36:157-168. doi:10.1007/s10858006-9077-y

Kazimierczuk K, Zawadzka A, Koźmiński W (2009) Narrow peaks and high dimensionalities: exploiting the advantages of random sampling. J Magn Reson 197:219-228. doi:10.1016/j.jmr.2009. 01.003

Kazimierczuk K, Zawadzka-Kazimierczuk A, Koźmiński W (2010) Non-uniform frequency domain for optimal exploitation of nonuniform sampling. J Magn Reson 205:286-292. doi:10.1016/j. jmr.2010.05.012

Kazimierczuk K, Misiak M, Stanek J, Zawadzka-Kazimierczuk A, Koźmiński W (2012) Generalized Fourier transform for non-uniform sampled data. Top Curr Chem 316:79-124. doi:10.1007/128

Kazimierczuk K, Stanek J, Zawadzka-Kazimierczuk A, Koźmiński W (2013) High-dimensional NMR spectra for structural studies of biomolecules. Chem Phys Chem 14:3015-3025. doi:10.1002/ cphc. 201300277
Mäntylahti S, Aitio O, Hellman M, Permi P (2010) HA-detected experiments for the backbone assignment of intrinsically disordered proteins. J Biomol NMR 47:171-181. doi:10.1007/ s10858-010-9421-0

Mobli M, Hoch JC (2008) Maximum entropy spectral reconstruction of non-uniformly sampled data. Concepts Magn Reson A Bridg Educ Res 32A:436-448. doi:10.1002/cmr.a.20126

Narayanan RL, Duerr HN, Bilbow S, Biernat J, Mendelkow E, Zweckstetter M (2010) Automatic assignment of the intrinsically disordered protein Tau with 441-residues. J Am Chem Soc 132:11906-11907. doi:10.1021/ja105657f

Nováček J, Zawadzka-Kazimierczuk A, Papoušková V, Žídek L, Šanderová H, Krásný L, Koźmiński W, Sklenár V (2011) 15D 13C-detected experiments for backbone assignment of unstructured proteins with a very low signal dispersion. J Biomol NMR 50:1-11. doi:10.1007/s10858-011-9496-2

Nováček J, Janda L, Dopitová R, Žídek L, Sklenář V (2013) Efficient protocol for backbone and side-chain assignments of large, intrinsically disordered proteins: transient secondary structure analysis of $49.2 \mathrm{kDa}$ microtubule associated protein $2 \mathrm{c}$. J Biomol NMR 56:291-301. doi:10.1007/s10858-013-9761-7

Nowakowski M, Saxena S, Stanek J, Żerko S, Koźmiński W (2015) Applications of high dimensionality experiments to biomolecular NMR. Prog Nucl Magn Res Spectrosc 34:93-158. doi:10.1016/j. pnmrs.2015.07.001

Orekhov VY, Jaravine VA (2011) Analysis of non-uniformly sampled spectra with multi-dimensional decomposition. Prog Nucl Magn Res Spectrosc 59:271-292. doi:10.1016/j.pnmrs.2011.02.002

Pantoja-Uceda D, Santoro J (2013) Direct correlation of consecutive $\mathrm{C}^{\prime}-\mathrm{N}$ groups in proteins: a method for the assignment of intrinsically disordered proteins. J Biomol NMR 57:57-63. doi:10.1007/s10858-013-9765-3

Piai A, Hošek T, Gonnelli L, Zawadzka-Kazimierczuk A, Koźmiński W, Brutscher B, Bermel W, Pierattelli R, Felli IC (2014) "CONCON" assignment strategy for highly flexible intrinsically disordered proteins. J Biomol NMR 60:209-218. doi:10.1007/ s10858-014-9867-6

Robin M, Delsuc M-A, Guittet E, Lallemand J-Y (1991) Optimized acquisition and processing schemes in three-dimensional NMR spectroscopy. J Magn Reson 92:645-650. doi:10.1016/00222364(91)90364-Y

Shaka AJ (1985) Composite pulses for ultra-broadband spin inversion. Chem Phys Lett 120:201-205. doi:10.1016/00092614(85)87040-8

Shaka AJ, Keeler J, Freeman R (1983) Evaluation of a new broadband decoupling sequence: WALTZ-16. J Magn Reson 53:313-340. doi:10.1016/0022-2364(83)90035-5

Stanek J, Koźmiński W (2010) Iterative algorithm of discrete fourier transform for processing randomly sampled NMR data sets. J Biomol NMR 47:65-77. doi:10.1007/s10858-010-9411-2

Stanek J, Augustyniak R, Koźminski W (2012) Suppression of sampling artefacts in high-resolution four-dimensional NMR spectra using signal separation algorithm. J Magn Reson 214:91-102. doi:10.1016/j.jmr.2011.10.009

Wright PE, Dyson HJ (1999) Intrinsically unstructured proteins: reassessing the protein structure-function paradigm. J Mol Biol 293:321-331. doi:10.1006/jmbi.1999.3110

Yao X, Becker S, Zweckstetter M (2014) A six-dimensional alpha proton detection-based APSY experiment for backbone assignment of intrinsically disordered proteins. J Biomol NMR 60:231-240. doi:10.1007/s10858-014-9872-9

Yoshimura Y, Kulminskaya NV, Mulder FAA (2015) Easy and unambiguous sequential assignments of intrinsically disordered proteins by correlating the backbone $15 \mathrm{~N}$ or $13 \mathrm{C}^{\prime}$ chemical shifts of multiple contiguous residues in highly resolved 3D 
spectra. J Biomol NMR 61:109-121. doi:10.1007/s10858-0149890-7

Zawadzka-Kazimierczuk A, Koźmiński W, Sanderova H, Krasny L (2012) High dimensional and high resolution pulse sequences for backbone resonance assignment of intrinsically disordered proteins. J Biomol NMR 52(4):329-337. doi:10.1007/s10858012-9613-x
Żerko S, Koźmiński W (2015) Six- and seven-dimensional experiments by combination of sparse random sampling and projection spectroscopy dedicated for backbone resonance assignment of intrinsically disordered proteins. J Biomol NMR 63:283-290. doi:10.1007/s10858-015-9987-7 